ORIGINAL ARTICLE

\title{
Evaluation of decompression safety in an occupational diving group using self reported diving exposure and health status
}

\section{J Doolette, D F Gorman}

See end of article for authors' affiliations

Correspondence to: Dr D Doolette, Anaesthesia \& Intensive Care, The University of Adelaide, Adelaide, Australia 5005; David.Doolette@ adelaide.edu.au

Accepted 17 September 2002
Background: Many occupational diving groups have substantially different diving patterns to those for which decompression schedules are validated.

Aims: To evaluate tuna farm occupational diving practice against existing decompression models and describe a method for collecting and modelling self reported field decompression data.

Methods: Machine readable objective depth/time profiles were obtained from depth/time recorders worn by funa farm occupational divers. Divers' health status was measured at the end of each working day using a self administered health survey that produces an interval diver health score (DHS) with possible values ranging from 0 to 30. Depth/time profiles were analysed according to existing decompression models. The contribution of diving exposure and between diver variability to DHS was evaluated using linear regression.

Results: The mean risk of decompression sickness was calculated as 0.005 (SD $0.003, n=383$ ). The mean DHS following diving was 3 (SD 2, n = 383) and following non-diving activities was 1 (SD 1 , $n=41$ ). After accounting for between diver variability in intercept, DHS was found to increase one unit for every $1 \%$ increase in the risk of decompression sickness.

Conclusions: A method has been established for the collection and analysis of self reported objective decompression data from occupational diving groups that can potentially be used as the basis for development of purpose designed occupational diving decompression schedules.
D ecompression sickness (DCS) is a significant health risk for occupational divers. The risk of DCS (pDCS) is minimised by use of a schedule of diving depths and durations and consequent decompression rates. Most decompression schedules are based on rudimentary models of gas kinetics, then tested under the intended operational conditions. pDCS can vary considerably with changes in diving conditions, ${ }^{1}$ and decompression schedules should be selected on proven reliability for similar exposures in a similar population. Instead, most decompression schedules are used generically such that uncertainty exists in the prediction of DCS. Most generically available decompression schedules originate from the military where it has been possible to conduct extensive human testing. This may be a problem for emerging occupational diving groups without specifically developed decompression schedules.

The tuna farming industry in Australia involves diving practices that are substantially different from those of conventional occupational and military divers. ${ }^{2}$ The Australian Standard for Occupational Diving ${ }^{3}$ requires the use of the Defence and Civil Institute of Environmental Medicine (DCIEM) standard air diving schedules ${ }^{4}$ for all occupational diving. These schedules are well tested and have a good operational record, but have not been evaluated in the unique context of tuna farm diving practices. Tuna farm diving practice involves extensive repetitive diving and multi-day diving (consecutive daily diving exposures); multi-day diving is avoided in military decompression schedule validation data. Consequently, the pDCS for tuna farm diving is unknown.

Widespread use of depth/time recorders by occupational divers makes accurate field data available from operational dives. DCS may not be reliably reported from dives conducted without medical supervision. However, the development of a scoring system for health status self assessment after diving provides a reliable measure of diving outcome. ${ }^{5}$ With careful audit, these data can be used to evaluate decompression practice against existing decompression models. Traditional deterministic decompression procedures, such as the DCIEM standard air diving, define acceptable diving schedules. More recent, probabilistic decompression models can assign pDCS to any depth/time profile. These latter models are produced using parameter estimation procedures to select decompression models of best fit to observed decompression data. ${ }^{6}$

The aims of this study are to describe tuna farm occupational diving practice and evaluate this practice against existing deterministic and probabilistic decompression models. A further aim is to describe and evaluate a method for collecting self reported field decompression data with the hypothesis that self reported health status is an indicator of decompression stress.

\section{METHODS}

\section{Data collection}

We used a prospective decompression data collection approach (approved by the University of Adelaide Human Ethics Committee and conducted in accordance with the "National statement on ethical conduct in research involving humans, 1999") that was based on machine readable objective depth/time profiles data and a novel self administered diver health survey. Machine readable depth/time profiles were obtained from diver carried depth/time recorders. These devices record gauge pressure (as depth of water) at fixed, device dependent intervals typically of 5 or 20 seconds.

Abbreviations: AIC, Akaike Information Criteria; DCIEM, Defence and Civil Institute of Environmental Medicine; DCS, decompression sickness; DHS, diver health score; DUR, dive duration; MSW, metres sea water; NUM, number of divers; pDCS, risk of DCS 
Development and validation of the diver health survey has been described in detail elsewhere. ${ }^{5}$ It is an inventory of nine standardised items and responses covering five symptoms of decompression illness (paraesthesia, rash, balance, vitality, and pain), five health status indicators (vitality, pain, physical functioning, role limitation, and health perception), and time of onset of symptoms, plus one free response, each item scored from 0 to 3. The resulting summed diver health score (DHS) can be analysed as interval data and is correlated with clinical decompression illness. ${ }^{5}$ The validated format of the diver health survey and scoring instructions are available from the corresponding author. The DHS was used as the outcome measure without any attempt to categorise dives as resulting in DCS or not.

The symptoms of DCS and of other decompression illnesses that arise from lung barotrauma are often indistinguishable. ${ }^{7}$ This is a limitation for validation of decompression models, since these can only predict DCS. However, lung barotrauma would not be expected as a common feature of the experienced occupational diver group in the present study.

Decompression data collection was organised through the tuna farm diving supervisors. Generally, for 1-2 month periods, dive team members completed a diver health survey at the end of any workday that included a diving exposure. Health surveys were returned in confidence by reply paid mail to our laboratory. Depth/time profiles were uploaded from recorders by either the diving supervisor or the farm secretarial staff, using the proprietary software for these devices. Depth/time profiles were copied to floppy disk and returned by reply paid mail. For baseline data, some divers completed health surveys following non-diving work activities.

Decompression data were managed using purpose designed, partially automated database and analysis applications programmed in our laboratory (Access 2000, Visual BASIC, Microsoft Corp., Redmond, WA; and Statistica BASIC, Statsoft, Inc., Tulsa, OK). Depth/time profile audit consisted of cross checking between daily log information (diver name, date, and number of dives) supplied separately by individual divers (health survey) and by company (depth/time profiles). Prior to analysis, to reduce computing time, raw depth/time profiles were reduced to a smaller number of depth/time nodes: distance between adjacent nodes was increased by sequential exclusion of intervening data points until reaching a threshold residual sum of squares between the excluded points and a line connecting the nodes. Raw depth/time profile and extracted nodes were visually inspected to exclude data errors. Repetitive dives (surface interval less than 18 hours) and surface intervals were combined into a single daily depth/ time profile.

From a larger database of health surveys and depth/time profiles, a diving data subset was identified comprising 383 verified decompression datum pairs, where both the full day's depth/time profiles and DHS were available (383 health surveys covering 527 dives conducted by 23 divers). Only divers contributing more than one DHS were included. Air was breathed throughout all dives. Each verified decompression datum was considered independent. In addition, nine of these divers contributed health surveys $(n=41)$ at the end of workdays during which they did not dive (non-diving data subset).

\section{Evaluation of decompression practice}

Each daily depth/time profile was checked for compliance with the DCIEM standard air diving schedules. The US Navy linear exponential kinetics probabilistic decompression model (USN93), which is a useful predictor of pDCS in military air diving, ${ }^{8}$ was used for reporting of pDCS for each daily depth/ time profile. An implementation of the USN93 model similar to that previously described, ${ }^{9}$ using published parameters, ${ }^{8}$ was written in the BASIC programming language (Visual BASIC, Microsoft Corp., Redmond, WA.) The pDCS was tracked over the daily depth/time profile and subsequent 18 hours.

\section{Modelling of DHS}

The DHS has been previously shown to correlate with clinical diagnosis for decompression illness. Here the DHS was used to measure decompression related health status among divers during normal diving operations; therefore, the contribution of diving exposure and interdiver variability to DHS in the absence of diagnosed decompression illness was evaluated by linear regression using the combined diving $(\mathrm{n}=383)$ and non-diving $(n=41)$ datasets. To accommodate possible interdiver variability we used a linear mixed effect modelling approach. The full model investigated was of the form:

$$
\begin{aligned}
& \mathrm{DHS}_{\mathrm{ij}}=\beta_{0 \mathrm{i}}+e_{\mathrm{i}}+\beta_{1} \mathrm{pDCS}_{\mathrm{ij}}+\beta_{2} \mathrm{DUR}_{\mathrm{ij}}+\beta_{3} \mathrm{MSW}_{\mathrm{ij}}+\beta_{4} \mathrm{NUM}_{\mathrm{ij}} \\
& +e_{\mathrm{ij}}
\end{aligned}
$$

which comprised the dependent variable DHS and fixed explanatory variables, pDCS, maximum daily depth in metres sea water (MSW), total daily dive duration in minutes (DUR), and the number of divers per day (NUM). The 23 divers were considered a random sample from a population where the intercept $\left(\beta_{0}\right)$ of the regression on the explanatory variables depends on the diver. Subscript i denotes divers, subscript $j$ denotes days, and $e$ denotes error.

Parameters of the regression models were estimated by maximising the likelihood. The likelihood is the joint probability density function of the observed values of the dependent variable given the respective regression model. To find the most parsimonious model, explanatory variables were manually removed from the full model and the resulting reduced models also fit to the data. Significant difference between nested models was evaluated by likelihood ratio test, $2\left(\mathrm{LL}_{\mathrm{p}}-\mathrm{LL}_{\mathrm{k}}\right) \cong \mathrm{X}_{\mathrm{p}-\mathrm{k}}^{2}$, where LL is the maximised log likelihood of the model and $\mathrm{p}$ and $\mathrm{k}$ are the number of parameters in the full and reduced model respectively $(\mathrm{p}>\mathrm{k})$. Models were selected according to minimum Akaike Information Criteria (AIC) which evaluate model fit to the data (maximised log likelihood, LL) and penalises for model complexity (number of estimated parameters, $p$ ), AIC $=-2 \mathrm{LL}+2 \mathrm{p}$. For the selected models, data were examined for influential values; these were considered outlier values with high leverage. Outliers were data with standardised residual more than 2 standard deviations from the mean. Leverage was taken as the diagonal of the hat matrix and values more than twice the mean were considered high.

All statistical calculations were performed using R software base package (version 1.4.1; The R Development Core Team, 2002) and the non-linear mixed effect package (version 3.1-23; Pinheiro J, Bates D, DebRoy S, Sarkar D, 2001).

\section{RESULTS}

\section{General diving practice}

Table 1 summarises the diving data subset. Repetitive dives were those separated by surface intervals greater than 10 minutes and less than 18 hours; the mean surface interval was 97 minutes ( $S D 56, \mathrm{n}=144$ ). There were 253 single dives, 116 double repetitive dive series, and 14 triple repetitive dive series. Repetitive diving exposures were generally deeper and longer than single dive exposure. Figure 1 shows typical depth/time profiles. Single dives were often long duration, multi-level dives for inspection and maintenance of the tow cages used to move tuna from the offshore fishing grounds or of the near-shore feeding pens. Repetitive dives were often short duration dives to remove dead tuna from the bottom of the feeding pens. Consecutive daily diving exposure (multiday diving) was common in tuna farm diving and occurred in 
Table 1 Diving data subset summary

\begin{tabular}{|c|c|c|c|c|c|}
\hline & Days & DHS & MSW & DUR & pDCS \\
\hline All dives & 383 & $3(2)$ & $18.0(3.4)$ & $32(25)$ & $0.005(0.003)$ \\
\hline Single dives & 253 & $3(2)$ & $17.4(3.4)^{*}$ & $29(20)^{*}$ & $0.004(0.002)^{*}$ \\
\hline Repetitive dives & 130 & $3(2)$ & $19.0(3.1)$ * & $38(32)$ * & $0.007(0.002)^{*}$ \\
\hline
\end{tabular}

Table 2 Diving data subset

\begin{tabular}{lclllll}
\hline Diver & Days & DHS & MSW & DUR & NUM & pDCS \\
\hline A & 13 & $2(1-5)$ & $20.4(10.7-22.3)$ & $46(10-190)$ & $1(1-2)$ & $0.005(0.002-0.009)$ \\
B & 18 & $5(2-14)$ & $20.7(10.5-21.7)$ & $27(8-127)$ & $1(1-2)$ & $0.005(0.002-0.010)$ \\
C & 13 & $4(1-12)$ & $20.9(11.4-22)$ & $40(9-127)$ & $1(1-2)$ & $0.006(0.001-0.014)$ \\
D & 17 & $3(2-4)$ & $20.9(10.5-22.1)$ & $30(9-174)$ & $2(1-3)$ & $0.005(0.002-0.012)$ \\
E & 10 & $3(2-3)$ & $19.7(10.4-21.6)$ & $26(8-105)$ & $1(1-3)$ & $0.004(0.002-0.008)$ \\
F & 8 & $2(1-6)$ & $19.6(19.1-20.5)$ & $28(11-44)$ & $2(1-3)$ & $0.007(0.004-0.009)$ \\
G & 17 & $3(1-6)$ & $19.2(5.1-19.9)$ & $25(11-51)$ & $1(1-3)$ & $0.004(0-0.013)$ \\
H & 67 & $2(0-6)$ & $19.1(1.9-22.4)$ & $25(3-66)$ & $1(1-3)$ & $0.004(0-0.011)$ \\
I & 60 & $3(0-12)$ & $18.9(3.3-20.3)$ & $28(6-81)$ & $1(1-2)$ & $0.004(0-0.011)$ \\
J & 3 & $4(3-5)$ & $20.8(20.7-21.4)$ & $39(31-43)$ & $2(2-3)$ & $0.009(0.009-0.012)$ \\
K & 5 & $0(0-1)$ & $19.9(19.7-20.0)$ & $37(22-61)$ & $2(2-3)$ & $0.008(0.006-0.012)$ \\
L & 34 & $2(1-6)$ & $19.2(3.8-20.5)$ & $20(4-148)$ & $1(1-2)$ & $0.006(0-0.013)$ \\
M & 4 & $2(2-2)$ & $19.0(18.7-19.4)$ & $47(22-59)$ & $1(1-1)$ & $0.006(0.002-0.01)$ \\
N & 18 & $2(1-3)$ & $20.2(19.5-21.0)$ & $16(10-38)$ & $1(1-2)$ & $0.006(0.004-0.009)$ \\
O & 9 & $2(0-2)$ & $15.8(15.0-19.5)$ & $38(21-62)$ & $1(1-2)$ & $0.004(0.002-0.007)$ \\
P & 9 & $4(2-7)$ & $15.0(13.5-16.5)$ & $31(22-36)$ & $1(1-1)$ & $0.002(0.001-0.004)$ \\
Q & 9 & $6(5-6)$ & $15.6(14.1-19.0)$ & $32(21-60)$ & $1(1-1)$ & $0.004(0.003-0.008)$ \\
R & 3 & $4(3-4)$ & $18.9(18.8-20.1)$ & $24(7-25)$ & $2(1-2)$ & $0.004(0.004-0.005)$ \\
S & 7 & $2(1-4)$ & $18.5(17.5-19.5)$ & $15(8-26)$ & $1(1-2)$ & $0.002(0.001-0.004)$ \\
T & 17 & $2(1-2)$ & $19.1(15.2-19.8)$ & $12(7-40)$ & $1(1-2)$ & $0.005(0.004-0.01)$ \\
U & 4 & $2(2-2)$ & $19.5(18.7-19.8)$ & $26(6-37)$ & $1(1-2)$ & $0.003(0.002-0.005)$ \\
V & 16 & $0(0-4)$ & $18.4(14.0-22.1)$ & $32(13-65)$ & $2(1-3)$ & $0.006(0.002-0.009)$ \\
W & 22 & $2(0-5)$ & $19.3(7.0-21.2)$ & $36(14-147)$ & $2(1-3)$ & $0.006(0.002-0.010)$ \\
\hline Results expressed as median (range). & & & \\
\hline & & & & & &
\end{tabular}

2 day $(\mathrm{n}=110), 3$ day $(\mathrm{n}=75), 4$ day $(\mathrm{n}=48)$, and 5 day $(\mathrm{n}=10)$ series. Table 2 gives the contribution of the individual divers to the diving data subset.
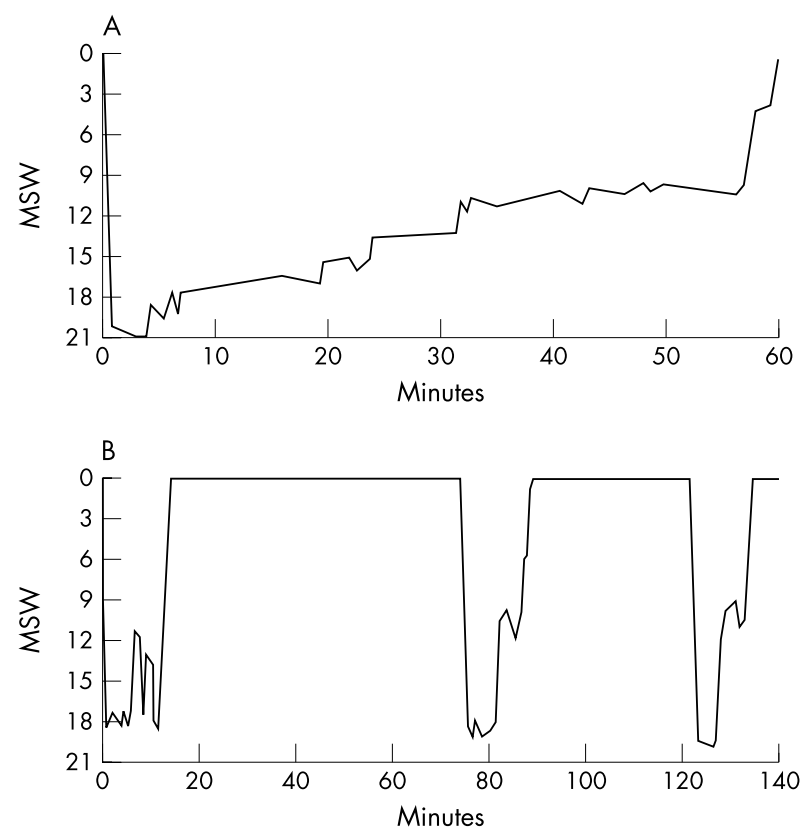

Figure 1 Typical depth/time profiles. (A) Single net inspection dive. (B) Repetitive "mortality" dives.

\section{Evaluation of decompression practice}

The mean interval between diving and health self assessment was 3 (SD 3, $\mathrm{n}=382$ ) hours, excluding the one diver health survey completed after a lengthy retrieval ( 144 hours) from a remote dive site for recompression therapy. This was the only diver who sought medical treatment for decompression illness $($ DHS $=14$ ). The mean DHS following diving was 3 (SD 2, $\mathrm{n}=346)$ and was not different between single and repetitive dives (table 1). Figure 2 shows the distribution of DHS following all diving activities. Nine of the divers completed health surveys following non-diving activities (table 3); the mean non-diving DHS was 1 (SD l, $\mathrm{n}=41$ ) but there were significant differences between individual divers' non-diving DHS (one way ANOVA, $\mathrm{df}=8, \mathrm{~F}=3.63, \mathrm{p}=0.004$ ).

Tuna farm divers are required to adhere to DCIEM standard air decompression schedules; only one daily exposure violated these schedules and the health outcome was unremarkable

\begin{tabular}{|lll|}
\hline Table 3 & \multicolumn{2}{l|}{ Non-diving data subset } \\
\hline Diver & Days & DHS \\
\hline A & 1 & 3 \\
C & 1 & 3 \\
H & 9 & $0(0-2)$ \\
I & 9 & $2(0-3)$ \\
L & 8 & $2(1-3)$ \\
M & 1 & 0 \\
R & 1 & 0 \\
V & 8 & $1(0-2)$ \\
W & 3 & $1(0-1)$ \\
\hline \multicolumn{2}{l}{ Results expressed as median (range). } \\
\hline
\end{tabular}




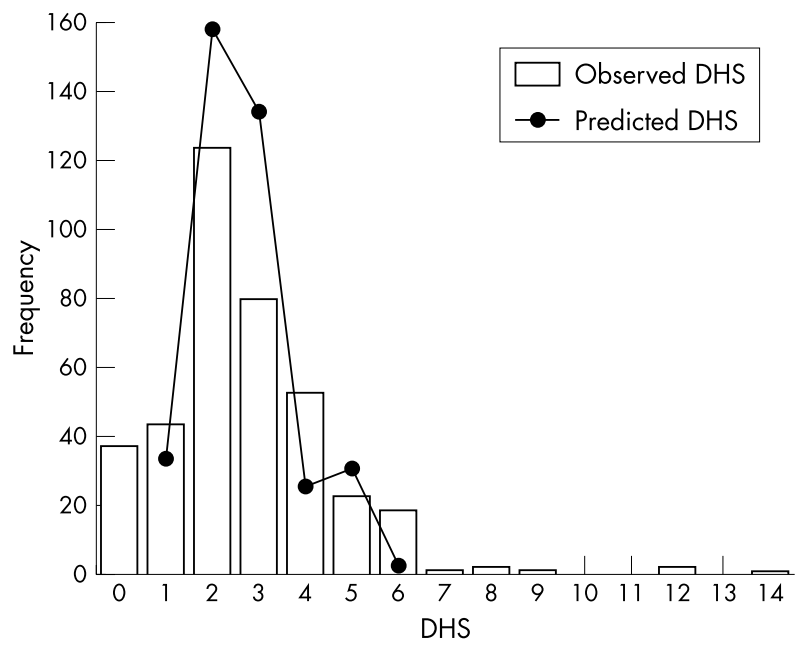

Figure 2 Frequencies of DHS measured following 383 diving days (bars) and DHS predicted from pDCS by model 4 (circles).

(DHS $=2$ ). A pDCS $\geqslant 0.0156$ is calculated from the USN93 model for the relevant no-decompression stop DCIEM standard air schedules. The mean pDCS for daily tuna farm daily depth/time profiles according to the USN93 model was 0.005 (SD 0.003) (see table 1). The highest risk exposures in the present data were typically short duration repetitive dives to depths in excess of $18 \mathrm{msw}(280 \mathrm{kPa})$ and with rapid decompression to the surface (fig $1 \mathrm{~B}$ ). The instantaneous risk evaluated in the USN93 model always declined to zero before the end of the 18 hour post-dive integration period and typically before 4 hours. This supports treatment of daily depth/time profiles as independent in the subsequent modelling procedures.

\section{Modelling of DHS}

The significant differences in non-diving DHS between divers (table 3) suggest there may be considerable inter-diver variability in all DHS. This was supported by factorial ordinary least squares regression with DHS as the dependent variable, pDCS as the independent variable, and diver as the between group factor; this produced highly variable parameters estimates between divers.

This inter-diver variability is accommodated in models compared in table 4; the variance of model intercept between divers (not shown) was 1.4 in each model. In the full model (1), explanatory variable MSW and NUM had non-significant parameter estimates and these were removed to produce a simpler model with similar fit to the data (model 2). Removal from model 2 of either DUR (model 3) or pDCS (model 4) resulted in significantly worse fit to the data. The null model (model 5) that comprised only the random intercept term produced significantly worse fit to the data than all other models.

Although inclusion of explanatory variable DUR in model 2 significantly improved fit to the data compared to model 4 , the parameter estimate of $\beta_{2}$ indicated only a unit change in DHS unit for every 167 minutes diving duration, unsubstantial in the context of mean dive duration of 32 minutes. The estimates of the common parameters were similar and the difference in fit was small, so the simpler model 4 was chosen for further investigation. In model 4 the estimate of parameter $\beta_{1}$ indicated approximately a unit change in DHS for every $1 \%$ change in pDCS. The intercept values ranged from 0.1 to 4.7 between divers. The four DHS values $>8$ were identified as outliers, but these did not have high leverage so were retained in the data. Figure 2 shows model 4 estimates of DHS: for the diving data subset the fitted values of DHS were rounded to the nearest integer and the frequency of each integer value (circles) superimposed on the frequency histogram of observed data (bars).

It was investigated whether there was a random effect of divers on both intercept and slope by allowing parameter $\beta_{1}$ to vary between divers, $\left(\beta_{1}+\eta_{\mathrm{i}}\right) \mathrm{pDCS}_{\mathrm{ij}}$. The improved fit to the data $(\mathrm{AIC}=1596, \mathrm{LL}=-792$ ) was not significantly different from model 4 (likelihood ratio 5.733, $\mathrm{df}=5,4, \mathrm{p}=0.0569$ ).

In this form of modelling the data are censored at the time the diver health survey is completed as symptoms of DCS may subsequently arise. However, this censoring is probably not severe as symptom onset occurs by three hours (mean interval between diving and health self assessment) in approximately $80 \%$ of cases of DCS. ${ }^{10}$ The contribution of this interval was examined by adding the fixed explanatory variable and parameter $\beta_{5}$ SINCE $_{\mathrm{ij}}$ to model 4 . The resulting model could only be fit to the diving data subset as there was no SINCE variable in the non-diving data subset. In addition the highly influential outlier value of DHS $=14$, SINCE $=144$ was excluded. Model $4($ AIC $=1417, \mathrm{LL}=-705)$ and the expanded model (AIC $=1419, \mathrm{LL}=-704$ ) were fit to this data subset and were not significantly different (likelihood ratio 0.921 , $\mathrm{df}=6,4, \mathrm{p}=0.337)$, and the estimate of parameter $\beta_{5}$ was not significant $(p=0.336)$, indicating that the interval was not important in this dataset.

\section{DISCUSSION}

The tuna farm diving practice reported here is relatively conservative as indicated by DHS skewed towards asymptomatic values and the general adherence to DCIEM standard air diving decompression schedules. The pDCS for tuna farm diving

\begin{tabular}{|c|c|c|c|c|c|c|c|c|}
\hline \multirow[b]{2}{*}{ Model } & \multirow[b]{2}{*}{ Variables } & \multirow{2}{*}{$\begin{array}{l}\text { Parameter } \\
\text { estimate (SE) }\end{array}$} & \multirow[b]{2}{*}{ df } & \multirow[b]{2}{*}{ AIC } & \multirow[b]{2}{*}{ LL } & \multicolumn{3}{|c|}{ Likelihood ratio } \\
\hline & & & & & & Test & Ratio & p value \\
\hline \multirow[t]{5}{*}{1} & Intercept & $1.79(0.35)$ & 7 & 1597 & -791.5 & & & \\
\hline & $\mathrm{pDCS}$ & $59.6(41.4)$ & & & & & & \\
\hline & DUR & $0.007(0.003)$ & & & & & & \\
\hline & MSW & $0.028(0.017)$ & & & & & & \\
\hline & NUM & $-0.076(0.177)$ & & & & & & \\
\hline \multirow[t]{2}{*}{2} & $\begin{array}{l}\text { Intercept } \\
\text { pDCS }\end{array}$ & $\begin{array}{l}2.03(0.31) \\
91.6(28.3)\end{array}$ & 5 & 1595 & -792.9 & $1 \vee 2$ & 2.6 & 0.2705 \\
\hline & DUR & $0.006(0.003)$ & & & & & & \\
\hline \multirow[t]{2}{*}{3} & Intercept & $2.41(0.28)$ & 4 & 1604 & -798.1 & $2 \vee 3$ & 10.4 & 0.0013 \\
\hline & DUR & $0.009(0.003)$ & & & & & & \\
\hline 4 & $\begin{array}{l}\text { Intercept } \\
\text { pDCS }\end{array}$ & $\begin{array}{l}2.15(0.30) \\
105(27.5)\end{array}$ & 4 & 1597 & -794.8 & $2 \vee 4$ & 3.8 & 0.0498 \\
\hline 5 & Intercept & $2.67(0.27)$ & 3 & 1610 & -802.0 & $4 \vee 5$ & 14.4 & 0.0001 \\
\hline
\end{tabular}


calculated according to the USN93 model was low compared to that calculated for no-decompression stop DCIEM standard air schedules; however, these latter figures assume that the full allowed time is spent at the maximum depth, a practice that is uncommon in the field. In addition, repetitive and multi-day diving was common and both may alter risk factors for DCS. The present conservative diving practice contrasts with anecdotal reports of high risk diving practice in the early days of the tuna farming industry, which are supported by the high incidence of decompression illness from that period, ${ }^{11}$ and is indicative of a recent positive reform of diving practice.

The USN93 model appears to be a reasonable predictor of tuna farm diving practice. The mean calculated pDCS of 0.005 equates to 1.9 incidents of DCS in 383 daily diving exposures and compares favourably with the one incident of treated DCS and the three additional DHS greater than 8 that may be untreated DCS. ${ }^{511}$ The military decompression data used to calibrate the USN93 model includes some diving practices relevant to tuna farming, but there are differences; in particular, multi-day diving is not included in the USN93 calibration data. For such reasons, occupational diving groups would benefit from specific decompression model development.

After accounting for inter-diver variability, the DHS is a useful measure of decompression related health outcome for field data collection and is positively correlated with decompression stress arising from normal diving operations in the absence of diagnosis of decompression illness. The methods for field decompression data collection and modelling reported here are promising, and the longer term prospect is to use such data to develop decompression schedules specific for tuna farm divers. This will require a more sophisticated modelling effort, possibly in combination with data from other sources, and continued field data collection.

In this regard, the self reporting methods described here are useful both in decompression data collection and subsequent analysis. First, field data can be easily collected, although careful procedures are necessary to ensure adequate data quality. Particular attention must be paid to the method of data audit to ensure full and accurate exposure data and to minimise selective reporting. In this context, crosschecking of data between divers and employers eliminated incomplete datasets and showed no indication of selective reporting from either source. In the absence of medical supervision, confidential self assessment of health status is probably the only valid method of measuring diving outcome. Divers may not report symptoms of DCS for a variety of reasons. ${ }^{12}$ Routine, confidential self reporting of health status can alleviate peer or economic pressures against reporting DCS and eliminates the need to evaluate the significance of symptoms in the decision to report. The DHS has been shown to be a valid and reliable measure of decompression related diving outcome. ${ }^{5}$

Secondly, interval measures such as the DHS have some statistical advantages compared to categorical data, particularly for small datasets. To avoid overfitting a binary dataset, the number of independent variables and fitted parameters in a model should be constrained to a fraction (for example, 0.1) of the number of the least frequent outcome $e^{13}$; thus to fit complex decompression models to health outcome categorised as the presence or absence of DCS requires a dataset with many incidents of DCS. An enormous data collection programme would be required to amass such a dataset from the field, where DCS is rare. On the other hand, use of an interval data DHS for each dive, as in the present study, has advantages in the modelling process because the number of fitted parameters is constrained by total sample size. Using these methods, a useful dataset can be acquired relatively easily. The present

\section{Main messages}

- Self assessment of health status can be used as an indicator of decompression outcome and correlates with decompression stress following normal diving operations.

- Recent tuna farm diving practice is conservative; nevertheless decompression sickness still occurs in spite of conservative diving practice.

\section{Policy implications}

- A relatively simple method exists for evaluating decompression practice in emerging diving industries.

methods cannot replace well controlled, medically supervised dives, which produce the primary data for decompression model development, but can complement this latter approach by making available field decompression data describing a variety of different diving practices.

\section{ACKNOWLEDGEMENTS}

This work was supported by a grant from WorkCover Corporation. We would like to thank the Tuna Boat Owners Association of Australia and the diving supervisors, divers, and secretarial staff from the tuna farming industry who participated in data collection.

\section{Authors' affiliations}

D J Doolette, Anaesthesia \& Intensive Care, The University of Adelaide, Australia

D F Gorman, Department of Medicine, University of Auckland, New Zealand; Anaesthesia \& Intensive Care, The University of Adelaide, Australia

\section{REFERENCES}

1 Leffler CT, White JC. Recompression treatments during the recovery of TWA Flight 800. Undersea Hyperb Med 1997;24:301-8.

2 Doolette DJ, Craig DS. Tuna farm diving in South Australia. South Pacific Underwater Medicine Society Journal 1999;29:1 15-17.

3 Standards Australia. Occupational diving operations-standard operational practice, AS/NZS 2299.1:1999.

4 DCIEM (Canada). DCIEM diving manual (air diving tables and procedures). Richmond, British Columbia: Universal Dive Techtronics, 1992.

5 Doolette DJ. Psychometric testing of a health survey for field reporting of decompression outcome. Undersea Hyperb Med 2000;27: 137-42.

6 Weathersby PK, Homer LD, Flynn ET. On the likelihood of decompression sickness. J Appl Physiol 1984;57:815-25.

7 Francis TJR, Smith DJ, eds. Describing decompression illness. Proceedings of the 42nd Undersea and Hyperbaric Medical Society Workshop, Oct 9-10 1990, Alverstoke, Gosport, Hampshire, UK. Bethesda, MD: Undersea and Hyperbaric Medical Society, 1991

8 Thalmann ED, Parker EC, Survanshi SS, et al. Improved probabilistic decompression model risk predictions using linear-exponential kinetics. Undersea Hyperb Med 1997;24:255-74.

9 Gerth WA, Vann RD. Probabilistic gas and bubble dynamics models of decompression sickness occurrence in air and $\mathrm{N}_{2}-\mathrm{O}_{2}$ diving. Undersea Hyperb Med 1997;24:275-92.

10 Elliott DH, Moon RE. Manifestations of the decompression disorders. In: Bennett PB, Elliott, DH, eds. The physiology and medicine of diving, 4th edn. London: W.B. Saunders Company, 1993

11 Whyte P, Doolette DJ, Gorman DF, et al. Positive reform of tuna farm diving in South Australia in response to a government intervention. Occup Environ Med 2001:58:124-8.

12 Weathersby PK, Survanshi SS. Data quality for decompression modeling. In: Sterk W, Hamilton RW, eds. Operational dive and decompression data: collection and analysis. Proceedings of the European Undersea biomedical Society Workshop, Aug 171990 Amsterdam, Netherlands. Amsterdam: Foundation for Hyperbaric Medicine, 1991:94-9.

13 Harrell FE, Lee KL, Mark DB. Multivariable prognostic models: issues in developing models, evaluating assumptions and adequacy, and measuring and reducing errors. Stat Med 1996;15:361-87. 